\title{
Consumo de fast food entre acadêmicos de Medicina
}

\author{
Fast food consumption among Medical students \\ Consumo de fast food entre estudiantes de Medicina
}

Recebido: 20/08/2021 | Revisado: 25/08/2021 | Aceito: 26/08/2021 | Publicado: 29/08/2021

\author{
Douglas Carlos Tuni \\ ORCID: https://orcid.org/0000-0001-7813-9641 \\ Universidade Comunitária da Região de Chapecó, Brasil \\ E-mail: tuni.douglas@outlook.com \\ Lucas Schenatto \\ ORCID: https://orcid.org/0000-0003-1891-7418 \\ Universidade Comunitária da Região de Chapecó, Brasil \\ E-mail: schenatto1999@gmail.com \\ Junir Antonio Lutinski \\ ORCID: https://orcid.org/0000-0003-0149-5415 \\ Universidade Comunitária da Região de Chapecó, Brasil \\ E-mail: junir@unochapeco.edu.br
}

\begin{abstract}
Resumo
O objetivo foi avaliar o perfil nutricional dos acadêmicos de Medicina da Universidade Comunitária da Região de Chapecó (Unochapecó) quanto ao consumo de fast foods. Estudo quantitativo qualitativo transversal descritivo, realizado por meio de questionário formulado na plataforma Google Forms, aplicado aos estudantes de Medicina da Unochapecó, no mês de março de 2019. Os dados obtidos foram organizados em uma planilha no sistema Microsoft Office Excel 2016 e PAST, versão 3.26, para análise das medidas de posição e dispersão, com auxílio de esquematizações de gráficos e tabelas. A amostra apresentou um $n=50$, com média de idade de 21,6 anos, peso médio de $62,4 \mathrm{~kg}$ e índice de massa corporal (IMC) de $21,9 \mathrm{~kg} / \mathrm{m}^{2}$, com majoritária participação dos acadêmicos do $3^{\circ}$ semestre. O consumo mais frequente de fast food foi duas vezes por mês (32\%), sendo a pizza e a batata frita os mais consumidos. Há maior predileção aos fast foods devido à rapidez, praticidade e o bom gosto ao paladar. Necessita-se de opções diversificadas para a alimentação nas universidades, com enfoque em alimentos saudáveis, como forma de prevenção de doenças crônicas não transmissíveis (DCNT), diminuindo os gastos públicos em medicamentos, internações e cirurgias.
\end{abstract}

Palavras-chave: Alimentação; Ciências de Saúde; Medicina.

\begin{abstract}
The objective was to evaluate the nutritional profile of the medical students of the Community University of the Region of Chapecó (Unochapecó) regarding the consumption of fast foods. This is a quantitative qualitative crosssectional study, through a questionnaire formulated in the Google Forms platform, applied to the students of Medicine of the Unochapecó, between March, 2019. The data obtained were organized in a spreadsheet in the Microsoft Office Excel 2016 system and PAST, version 3.26, to analyze the position and dispersion measures, with the help of graphs and tables. The sample had a $\mathrm{n}=50$, with a mean age of 21.6 years, average weight of $62.4 \mathrm{~kg}$ and BMI of $21.9 \mathrm{~kg} / \mathrm{m}^{2}$, with the majority participation of students from the 3rd semester. The highest frequency of fast food consumption was twice a month $(32 \%)$, and the most ingested foods were pizza and chips. There is a greater preference for fast foods due to its speed, practicality and good taste. There is a need for diversified food options in universities, focusing on healthy foods, as a form of prevention of chronic non-communicable diseases (NCDs), reducing public spending on medicines, hospitalizations and surgeries.
\end{abstract}

Keywords: Feeding; Health science; Medicine.

\section{Resumen}

El objetivo fue evaluar el perfil nutricional de los estudiantes de medicina de la Universidad Comunitaria de la Región Chapecó (Unochapecó) en relación al consumo de fast food. Estudio cuantitativo descriptivo transversal cuantitativo, realizado a través de un cuestionario formulado en la plataforma Google Forms, aplicado a estudiantes de medicina de Unochapecó, en marzo de 2019. Los datos obtenidos fueron organizados en el sistema Microsoft Office Excel 2016 y PAST, versión 3.26, para análisis de medidas de posición y dispersión, con la ayuda de diagramas de gráficos y tablas. La muestra presentó un $\mathrm{n}=50$, con una edad media de 21,6 años, peso medio de $62,4 \mathrm{~kg}$ e índice de masa corporal (IMC) de $21,9 \mathrm{~kg} / \mathrm{m} 2$, con la participación mayoritaria de estudiantes del 3er semestre. El consumo más frecuente de comida rápida fue dos veces al mes (32\%), siendo la pizza y las papas fritas las más consumidas. Existe una mayor predilección por las comidas rápidas por su rapidez, practicidad y buen gusto en el paladar. Existe la 
necesidad de opciones diversificadas de alimentación en las universidades, con un enfoque en alimentos saludables, como una forma de prevenir las enfermedades crónicas no transmisibles (ENT), reduciendo el gasto público en medicamentos, hospitalizaciones y cirugías.

Palabras clave: Alimentos; Ciencias de la Salud; Medicamento.

\section{Introdução}

O ingresso na universidade é um período de transição da adolescência para a vida adulta, com traços de independência e escolhas, apresentando reflexos a longo prazo (Bernardo et al., 2017). Desse modo, busca-se entender o perfil nutricional dos acadêmicos de medicina da Unochapecó, atentando-se para o consumo de fast food, visto que a alimentação dos universitários está calcada na ingesta de doces, alimentos gordurosos e baixa ingestão de frutas e hortaliças (Aquino, Pereira \& Reis, 2015) .

A prática de uma alimentação inadequada, com ingestão excessiva de ultraprocessados é um fator que predispõe à obesidade e doenças crônicas não transmissíveis, tais como hipertensão arterial, diabetes, anemia e hipovitaminose (Kapinos, Yakusheva \& Eisenberg, 2014; Santos, Costa \& Ferreira, 2021). Assim sendo, o estudo acerca das preferências alimentares dos acadêmicos se torna relevante, visto que a expectativa de vida vem aumentando, a população está apresentando cada vez mais morbidades e o sistema único de saúde (SUS) vem enfrentando dificuldades devido aos gastos com tratamento de doenças, ao invés da prevenção destas (Matos et al., 2019).

Nessa mesma perspectiva, faz-se necessário avaliar as medidas antropométricas dos acadêmicos que consomem fast foods. O aumento do IMC aos níveis da obesidade predispõe a elevação de triglicérides, LDL-c, colesterol total e a presença de alterações metabólicas, caracterizando a Síndrome Metabólica, muito por conta da má alimentação aliada ao ambiente em que se está inserido (Alimoradi et al., 2017; Gruinset et al., 2019).

Alimoradi et al. (2017) afirmam que a vida é influenciada pelo estilo de vida adotado pelo indivíduo. Além disso, os genes das doenças podem ser acionados ou não, conforme o padrão alimentar, realização de atividades físicas e a influência ambiental. Não obstante, Silva \& Mendes (2019) relataram que os fatores alimentares e a própria formação do corpo humano iniciam na infância, sofrendo adaptações de acordo com as crenças familiares e fatores socioculturais.

Neste contexto, objetivou-se avaliar o perfil nutricional dos acadêmicos de Medicina da Universidade Comunitária da Região de Chapecó (Unochapecó) quanto ao consumo de fast foods, buscando compreender a frequência de consumo, quais os alimentos preferidos, além de correlacionar com dados antropométricos e verificar as possíveis alterações decorrentes dos hábitos alimentares.

\section{Metodologia}

Trata-se de um estudo quantitativo e qualitativo transversal descritivo sobre o perfil dos acadêmicos de medicina da Unochapecó quanto ao consumo de fast foods.

A distinção principal na abordagem do quantitativo e qualitativo, nas ciências, está na forma de representar o real. Isto pode se dar através de números, caracterizando-a quantitativamente, ou por meio de aspectos subjetivos, singularizando o aspecto qualitativo do estudo (Ferreira, 2015).

O estudo foi desenvolvido junto aos estudantes do curso de medicina da Unochapecó, que responderam a um questionário semiestruturado de autoria dos próprios pesquisadores, utilizando-se a base na plataforma Google Forms. A coleta foi realizada durante o mês de março de 2019.

A coleta dos dados incluiu os acadêmicos de medicina que se autodeclaram consumidores de fast foods e que responderam voluntariamente ao questionário. $\mathrm{O}$ critério de exclusão utilizado deu-se por base naqueles que não se declararam consumidores de fast foods. 
O questionário contemplou variáveis sociodemográficas como o sexo, idade e semestre do curso do universitário. Em uma segunda etapa contemplou informações sobre as medidas antropométricas (peso, altura e IMC) dos acadêmicos. Buscou verificar o se o indivíduo consome fast food e a frequência de consumo do mesmo. Atentou-se para quais são os fast foods mais consumidos pelos acadêmicos e para as razões que levam o estudante a consumi-los. Por fim, contemplou questões para entender com quem o acadêmico costuma comer.

Os dados obtidos foram organizados em uma planilha no sistema Microsoft Office Excel 2016 para quantificação e análise dos resultados a fim de possibilitar a discussão dos dados coletados. Os dados analisados quanto às medidas de posição e de dispersão, com o auxílio do software PAST. Foram utilizados testes paramétricos e não paramétricos, tais como o chiquadrado, Kruskal-Wallis, teste t, Mann-Whitney e correlação de Pearson. Os resultados foram organizados em gráficos e tabelas com o intuito de elucidar as informações obtidas.

O presente estudo está de acordo com as orientações éticas de pesquisa que resguardam o respeito às pessoas, conforme estabelece a resolução 466/12 do Conselho Nacional de Saúde. Participaram acadêmicos, maiores de 18 anos, que voluntariamente concordaram em responder ao questionário. Concomitantemente, os dados coletados estão de posse dos autores, que manterão as identidades dos voluntários sob sigilo.

\section{Resultados}

O perfil da amostra de consumidores de fast food foi apresentado na Tabela 1. Observou-se uma maior porcentagem de consumidores do sexo feminino, matriculados no $3^{\circ}$ período do curso. O uso do teste $t$ apontou diferença no peso dos estudantes em relação à idade $(\mathrm{t}=22,13 ; \mathrm{p}<0,001)$.

Tabela 1- Perfil dos estudantes consumidores de fast foods, acadêmicos do curso de Medicina de uma universidade catarinense, Chapecó, 2019.

\begin{tabular}{lrr}
\hline Sexo & Contagem & Percentual \\
\hline Masculino & 14 & $28 \%$ \\
Feminino & 36 & $72 \%$ \\
\hline Período & Contagem & Percentual \\
\hline $1^{\circ}$ período & 18 & $36 \%$ \\
$3^{\circ}$ período & 22 & $44 \%$ \\
$5^{\circ}$ período & 10 & $20 \%$ \\
\hline Idade & Média & DP \\
\hline Masculino & 21,65 & 4,37 \\
Feminino & 21,67 & 4,33 \\
\hline
\end{tabular}

Fonte: Os autores (2019).

Quanto ao IMC, não foi encontrada diferença significativa entre os sexos (U=169,5; p>0,05) (Figura 1). Não foi encontrada correlação entre o IMC e os períodos do curso ( $r=0,17 ; \mathrm{p}>0,05)$. O perfil dos acompanhantes não diferiu quanto ao IMC dos estudantes $(\mathrm{Hc}=5,79 ; \mathrm{p}>0,05)$, assim como a frequência de consumo $(\mathrm{Hc}=5,52 ; \mathrm{p}>0,05)$. 
Figura 1- Comparativo do IMC, segundo o sexo, entre acadêmicos do curso de Medicina de uma universidade catarinense, Chapecó, 2019.

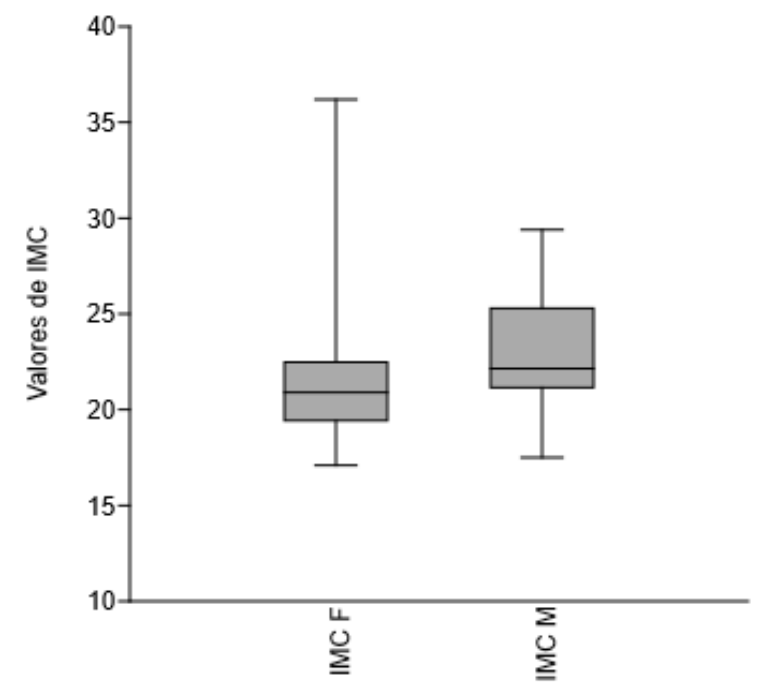

Fonte: Os autores (2019).

Os alimentos mais frequentemente relatados quanto ao consumo foram a pizza (92\%), seguida pela batata frita (72\%), sanduíche (68\%), hambúrguer (64\%) e macarrão instantâneo (36\%) (Tabela 2). Apenas o consumo de macarrão instantâneo apresentou relação com o IMC (U=157,5; p<0,05).

Tabela 2 - Relação entre o IMC e o perfil de consumo de fast food de acadêmicos do curso de Medicina de uma universidade catarinense, Chapecó, 2019.

\begin{tabular}{lcccc}
\hline Perfil do consumo & $\mathbf{n}$ & Percentual & Teste & p \\
\hline Consome hambúrguer & 32 & 64 & $\mathrm{U}=277$ & $>0,05$ \\
Não consome hambúrguer & 18 & 36 & & $<0,05$ \\
\hline Consome macarrão instantâneo & 18 & 36 & $\mathrm{U}=157,5$ & \\
Não consome macarrão instantâneo & 32 & 64 & & $>0,05$ \\
\hline Consome batata frita & 36 & 72 & $\mathrm{U}=238$ & $>0,05$ \\
Não consome batata frita & 14 & 28 & $\mathrm{U}=68,5$ & \\
\hline Consome pizza & 46 & 92 & & $>0,05$ \\
Não consome pizza & 4 & 8 & $\mathrm{U}=246,5$ & \\
\hline Consome sanduíche & 34 & 68 & 32 & \\
Não consome sanduíche & 16 & & \\
\hline
\end{tabular}

Fonte: Os autores (2019).

Quanto ao consumo de fast food, observou-se associação entre o consumo de hambúrguer o sexo $(\chi=4,27 ; \mathrm{p}<0,05)$.

A maior frequência de consumo de fast food ocorre duas vezes por mês (32\%), seguida de uma vez por mês e uma vez por semana, ambos com 30\%. A menor frequência de consumo é duas vezes por semana (8\%) (Tabela 4). 
Tabela 3 - Relação entre o sexo com o consumo de fast foods, entre acadêmicos do curso de Medicina de uma universidade catarinense, Chapecó, 2019.

\begin{tabular}{|c|c|c|c|c|}
\hline Sexo & Consome & Não consome & Teste & $\mathbf{p}$ \\
\hline \multicolumn{5}{|c|}{ Consumo de hambúrguer } \\
\hline Masculino & $8 \%$ & $20 \%$ & $\chi=4,27$ & $\mathrm{p}<0,05$ \\
\hline Feminino & $44 \%$ & $28 \%$ & & \\
\hline \multicolumn{5}{|c|}{ Consumo de macarrão instantâneo } \\
\hline Masculino & $6 \%$ & $22 \%$ & $\chi=1,79$ & $\mathrm{p}>0,05$ \\
\hline Feminino & $30 \%$ & $42 \%$ & & \\
\hline \multicolumn{5}{|c|}{ Consumo de batata frita } \\
\hline Masculino & $24 \%$ & $4 \%$ & $\chi=1,81$ & $\mathrm{P}>0,05$ \\
\hline Feminino & $48 \%$ & $24 \%$ & & \\
\hline \multicolumn{5}{|c|}{ Consumo de pizza } \\
\hline Masculino & $26 \%$ & $2 \%$ & $\chi=0,01$ & $\mathrm{p}>0,05$ \\
\hline Feminino & $66 \%$ & $6 \%$ & & \\
\hline \multicolumn{5}{|c|}{ Consumo de sanduíche } \\
\hline & $14 \%$ & $14 \%$ & $\chi=2,89$ & $\mathrm{p}>0,05$ \\
\hline & $18 \%$ & $54 \%$ & & \\
\hline
\end{tabular}

Fonte: Os autores (2019).

Tabela 4 - Frequência de consumo de fast food de acadêmicos de medicina de uma Universidade do Oeste de Santa Catarina (Chapecó, 2019).

\begin{tabular}{lcc}
\hline Consumo de fast food & Frequência & Porcentagem \\
\hline Uma vez por semana & 15 & 30,00 \\
Duas vezes por semana & 4 & 8,00 \\
Uma vez por mês & 15 & 30,00 \\
Duas vezes por mês & 16 & 32,00 \\
\hline Total & $\mathbf{5 0}$ & $\mathbf{1 0 0 , 0}$ \\
\hline
\end{tabular}

Fonte: Os autores (2019).

Acerca das razões que levam os acadêmicos a consumirem fast food, observou-se predominância de respostas de agrado ao paladar (40\%), seguido da rapidez e praticidade (28\%), falta de tempo (15\%) e preço acessível (8\%).

A alimentação rápida, muitas vezes, ocorre com o acompanhamento de amigos ou familiares. Os acadêmicos costumam consumir fast food com os amigos (50\%), seguida de familiares (34\%), sozinho ou com outros acompanhantes, ambos com $8 \%$. Contudo, a companhia não interfere na alimentação de fast food $(\chi=2,82 ; \mathrm{p}>0,05)$.

\section{Discussão}

O sexo feminino apresenta hábitos alimentares mais saudáveis como afirma Teleman et al. (2015), analisando os costumes alimentares de estudantes italianos. Na mesma direção, Sousa, José \& Barbosa (2013) encontraram os mesmos resultados, quando avaliaram as condutas negativas à saúde em estudantes universitários brasileiros, no estado da Bahia. 
Contudo, no presente estudo, evidenciou-se maior consumo de fast food entre mulheres, em contrapartida ao observado na literatura. Nesse mesmo sentido, Feitosa et al (2010) apontam um predomínio absoluto das mulheres em cursos da área da saúde, e isto tende a ter um impacto maior no cuidado alimentar, mas também na constituição de amostras.

Quanto a importância dos cuidados em saúde, o IMC dos estudantes não apresentou relação com a idade, sexo, período do curso e frequência de consumo. Fraser et al. (2011) apontam que o IMC tende a aumentar em crianças, contudo, não se mantém na adolescência e idade adulta. A homogeneidade da amostra pode explicar a similaridade nos valores do IMC encontrado.

Considerando-se que a amostra cursa um curso de ensino superior, a interação escolaridade e excesso de peso não é conclusiva, assim como apontou o estudo de Silva (2011). Graduar-se em um curso voltado à saúde não é fator protetivo contra a má-alimentação e hábitos não-saudáveis (Bernardo et al., 2017). Os resultados encontrados para o IMC da amostra tenderam para uma condição de eutrofia, indicando boa qualidade do estado de saúde dos estudantes.

A variação no peso dos estudantes participantes da pesquisa pode ser explicado pelo padrão mundial de consumo em países em desenvolvimento (Botelho \& Lameiras, 2018). A ingesta regular de fast food aumenta as chances de desenvolver obesidade (El-Qudah, 2014), embora isso não tenha sido constatado na amostra.

Sobre a frequência do consumo de fast food, houve uma regularidade maior na ingesta duas vezes por mês (32\%), seguido de uma vez por mês e uma vez por semana, ambos com 30\%). O estudo de Dias \& Gonçalves (2009) apontou que a maior frequência de consumo de alimentos desta natureza foi de uma vez por semana, diferentemente dos resultados do presente estudo. A menor frequência de consumo constatada neste estudo pode também caracterizar uma alimentação mais saudável e explicar o IMC eutrófico.

Os motivos pelos quais os acadêmicos escolhem consumir fast foods baseiam-se no bom grado ao paladar e na rapidez e praticidade. O estudo de Deliens et al. (2014) lista alguns facilitadores quanto à ingesta de alimentos não-saudáveis, entre eles a falta de tempo e o fácil acesso a esse nicho alimentar. Basaglia, Marques \& Benatti (2015) ainda acrescentam aspectos que propiciam uma aceitação alimentar, tais como o aroma, aparência, cor e textura.

O estudo de Dias \& Gonçalves (2009) estimou o consumo diário de produtos com alto teor de ácidos graxos trans, em indivíduos adultos, estando presente o hambúrguer e a batata frita. No presente estudo, há um elevado consumo de batata frita (72\%), seguido da ingestão de hambúrguer é a segunda menor (64\%). Essa preferência alimentar encontrada está, portanto, em acordo com a literatura e reflete aspectos da cultura ocidental, também presente no Brasil.

Quanto ao consumo de pizza, predominante na amostra (92\%), observou-se um acompanhamento da tendência brasileira a ingestão desse alimento, já que o país é o segundo mercado consumidor desse alimento no mundo. Esse resultado está em consonância com o estudo de Anjos et al. (2011), onde os consumidores que estão no ensino superior também levam em consideração o preço acessível do produto.

Cabe ressaltar que a alimentação interfere no desempenho acadêmico, visto que propicia a capacidade de concentração e aprendizagem (Nascimento et al., 2016). Ademais, o consumo de fast foods tende a perpertuar-se durante o ensino superior, mantendo esse hábito pelo resto da vida (Alves \& Precioso, 2017). Para tanto, é necessária a inclusão em ambiente universitário de locais que ofereçam uma alimentação balanceada, acessível, rápida e prática, justamente os parâmetros levantados pelos participantes da pesquisa.

De modo geral, as relações sociais e o ambiente familiar predispõem ao consumo de fast food (Alimoradi et al., 2017; Silva \& Mendes, 2019). No presente estudo, notou-se uma maior influência dos amigos e familiares quanto à ingesta de alimentos ultraprocessados, assim como aponta o estudo de Al-sheyab et al. (2019), que analisa o comportamento de adolescentes jordanos e o estilo de vida, relacionados à alimentação e exercícios físicos. 


\section{Conclusão}

O ingresso no ensino superior, seguido da passagem da adolescência para a vida adulta está relacionado às mudanças nos hábitos alimentares, em detrimento de um menor tempo para se alimentar, uma habitação longe dos pais e um gasto financeiro com os estudos, que restringe o acadêmico nas escolhas alentares saudáveis.

Como visto, há uma preferência maior em consumir alimentos processados, rápidos e palatáveis, deficitários em nutrientes. Desse modo, necessita-se a implementação de restaurantes, em universidades e nas proximidades, que propiciem uma alimentação saudável, rápida e acessível, financeiramente e temporariamente, dado a celeridade da vida acadêmica.

Assim sendo, estimular-se-á o consumo saudável e consciente entre os acadêmicos, visando prevenir a obesidade e a consequente evolução para Doenças Crônicas Não-Transmissíveis (DCNT), diminuindo os gastos públicos com medicação, internações e cirurgias.

Considerando o processo dinâmico que envolve o ensino superior e o competitivo contexto que envolve a formação em Medicina, recomenda-se estudos adicionais sobre o tema desta pesquisa. Estudos que possam monitorar as variações do padrão encontrado nesta pesquisa, tanto espacialmente, quanto temporalmente.

\section{Agradecimentos}

À Universidade Comunitária da Região de Chapecó pelo apoio à pesquisa.

\section{Referências}

Al-sheyab, N. A., Alomari, M. A., Hayajneh, A. A., \& Shah S. (2019). Attitudes and perceived barriers toward healthy lifestyle behaviors in Jordanian adolescents: a developing country perspective. Adolescent Health, Medicine and Therapeutics 10, 39-47. http://dx.doi.org/10.2147/AHMT.S181001

Alimoradi, F., Jandaghi, P., Khodabakhshi, A., Javadi, M., \& Moghadam, S. A. H. Z. (2017). Breakfast and fast food eating behavior in relation to sóciodemographic differences among school adolescents in Sanandaj Province, Iran. Electronic Physician Journal. 9(6), 4510-4515. http://dx.doi.org/10.19082/4510

Alves, R., \& Precioso, J. (2017). Hábitos alimentares dos/as estudantes do ensino superior. Revista de Estudios e Investigación en Psicología y Educación. 14, 239-244. https://doi.org/10.17979/reipe.2017.0.14.2974

Anjos, G. C. S. C., Andrade, A. A. C., Andrade, A. I. S., Pires, L. M., Pereira, K. N., \& Pinheiro, T. G. R. (2011). O perfil do consumidor de pizza na cidade de Juazeiro - BA. Intercom,.

Aquino, J. K., Pereira, P., \& Reis, V. M. C. P. (2015). Hábito e consumo alimentar de estudantes do curso de nutrição das faculdades de Montes Claros Minas Gerais. Revista Multitexto. 3(1), 82-88.

Basaglia, P., Marques, A. S., \& Benatti L. (2015). Aceitação da merenda escolar entre alunos da rede estadual de ensino da cidade de Amparo-SP. Saúde em Foco. 7, 126-138.

Bernardo, G. L., Jomori, M. M., Fernandes, A. C., \& Proença, R, P. C. (2017). Food intake of university students. Brazilian Journal of Nutrition. 30(6), 847865. https://doi.org/10.1590/1678-98652017000600016.

Botelho, G., \& Lameiras J. (2018). Adolescente e obesidade: considerações sobre a importância da educação alimentar. Acta Portuguesa de Nutrição. 15, 3035. http://dx.doi.org/10.21011/apn.2018.1506

Deliens, T., Clarys, P., Bourdeaudhuij, I., \& Deforche, B. (2014). Determinants of eating behaviour in university students: A qualitative study using focus group discussions. BMC Public Health. 14(53), 1-12. https://doi.org/10.1186/1471-2458-14-53.

Dias, J. R, \& Gonçalves, E. C. B. A. (2009). (2009). Avaliação do consumo e análise da rotulagem nutricional de alimentos com alto teor de ácidos graxos trans. Ciência e Tecnologia de Alimentos. 29(1), 177-182.

El-Qudah, J. M. (2014). Food habits and physical activity patterns among Jordanian adolescentes aged 11-18 years. World Applied Sciences Journal. 29(10), 1213-1219. http://dx.doi.org/10.5829/idosi.wasj.2014.29.10.1972

Feitosa, E. P. S., Dantas, C. A. O., Andrade-Wartha, E. R. S., Marcellini, P. M., \& Mendes-Netto, R.S. (2010). Hábitos alimentares de estudantes de uma universidade pública. Alimentos e Nutrição Araraquara. 21(2), 225-30

Ferreira, C. A. L. ( 2015). Pesquisa quantitativa e qualitativa: perspectivas para o campo da educação. Revista Mosaico. 8(2), 173-182. http://dx.doi.org/10.18224/mos.v8i2.4424

Fraser, L. K., Edwards, K. L., Cade, J. E., \& Clarke, G. P. (2011). Fast food, other choices and body mass index in teenagers in the United Kingdom (ALSPAC): a structural equation modelling approach. International Journal of Obesity. 35, 1325-1330. http://dx.doi.org/10.1038/ijo.2011.120 
Research, Society and Development, v. 10, n. 11, e212101119651, 2021

(CC BY 4.0) | ISSN 2525-3409 | DOI: http://dx.doi.org/10.33448/rsd-v10i11.19651

Gruinset, A. C., Cook, A. S., Conti, J., Gwizd, M., \& Farinelli, M. A. (2019) “Doing a good thing for myself”: a qualitative study of young adults' strategies for reducing takeaway food consumption. BMC Public Health. 19, 525-537. https://doi.org/10.1186/s12889-019-6731-3

Kapinos, K. A., Yakusheva, O., \& Eisenberg, D. (2014). Obesogenic environmental influences on young adults: Evidence from college dormitory assignments. Economics and Human Biology. 12, 98-109. https://doi.org/10.1016/j.ehb.2013.05.003.

Matos, C. H., Cruz, F. A. Z., Pereira, J. A. O., \& Henn, R. (2019). Tendência de consumo de alimentos industrializados por portadores de doenças crônicas não transmissíveis. Revista Contexto \& Saúde. 19(37), 37-43. https://doi.org/10.21527/2176-7114.2019.37.37-43

Nascimento, M. C., Oliveira, J. B., Fontan, G. C. R., \& Silva, M. V. (2016). Avaliação da adesão e aceitabilidade dos cardápios do Programa de Alimentação Escolar em escolas municipais de Itapetinga - BA: indicadores de desperdício de alimentos. Revista Eletrônica em Gestão, Educação e Tecnologia Ambiental. 20(1), 73-85.

Santos, A. M., Costa, E. F., \& Ferreira, J. C. S. (2021). Avaliação dos cardápios quanto a composição nutricional de escolas municipais da área urbana e rural da cidade de Manaus. Research, Society and Development. 10(7), e25810716687. http://dx.doi.org/10.33448/rsd-v10i7.16687.

Silva, H. P. C. (2011). Você é o que você come? Um estudo sobre o perfil de sobrepeso e obesidade no Brasil e os determinantes do IMC. Insper

Silva, F. R., \& Mendes, A. E. P. (2019).Percepção corporal, estado nutricional e comportamentos de risco para os transtornos alimentares. Revista Contexto \& Saúde. 19(36), 27-32. https://doi.org/10.21527/2176-7114.2019.36.27-32

Sousa, T. F., José, H. P. M., \& Barbosa, A. R. (2013). Condutas negativas à saúde em estudantes univer-sitários brasileiros. Ciência \& Saúde Coletiva. 18(12), 3563-75. http://dx.doi.org/10.1590/S1413-81232013001200013

Teleman, A. A., Waure, C., Soffiani, V., Poscia, A., \& Di Pietro, M. L. (2015). Nutritional habits in Italian university students. Annali dell'Istituto Superiore di Sanità. 51(2), 99-105. https://doi.org/10.4415/ANN_15_02_05 